\title{
Asymptotics and Quotient Spaces of Solutions of Operator-Difference Equations and Differential Equations with Small Delay
}

\author{
Zhumagul Zheentaeva \\ Kyrgyz-Uzbek University, Osh, Kyrgyzstan
}

Article History: Received: 10 November 2020; Revised 12 January 2021 Accepted: 27 January 2021; Published online: 5 April 2021

\begin{abstract}
Formerly, in order to conduct the in-depth study of differential equations with delay, the author proposed the method of splitting the solution space reducing such equations to the systems of operator-difference equations. Using this method, the author assumed new conditions, i.e. the absolute domains for coefficients sufficient for the existence of special (slowly changing) solutions, and proved the presence of approximating and asymptotically approximating properties in them, as well as the asymptotic one-dimensional space of solutions of the initial problems for linear scalar differential equations with insignificantly retarded argument and the corresponding operator-difference equation systems (special solutions correspond, to the solutions with a slowly changing first component and a relatively small second component). For the purposes of the single-point representation of the obtained results and other data related to the theory of dynamic systems (the distance between the solution values tends to zero alongside the unlimited increase in argument), throughout this research paper the author uses the concept of the asymptotic equivalence of solutions for dynamic systems, as it was introduced by the author in their previous research. In order to shape the new mathematical objects, the concept of asymptotic Hausdorff equivalence of solutions for dynamic systems is introduced (the distance between solution values tends to zero with unlimited increase in argument of one solution and monotonic transformation of argument of another solution).
\end{abstract}

Keywords: Finite-Difference Equation, Differential Equation with Retarded Argument, System of Equations, Special Solution, Initial Problem, Asymptotic

\section{Introduction}

This research paper discusses the dichotomy of solutions of the initial problems for dynamic systems (in the broad sense), and consequences thereof. It is noteworthy, that for solutions of linear autonomous (and with certain reservations, for periodic functions-coefficients) dynamic systems, the subject matter of the solution space structure is reduced to the research study of typical algebraic equations. Consequently, further herein the substantially non-autonomous equations shall be in focus.

In order to provide solution of the initial problem for a linear differential equation with a limited retarded argument, some sources, including the book by Myshkis (1972) and research papers by Driver et al. (1973) and Driver (1976), discuss the conditions when there exists such one-dimensional sub-space of solutions (referred to as special), so that any solution tends to increase its argument towards one of the special solutions. The review of such academic sources is given in the research paper by Pankov (1977a). Another research paper by Pankov (1977b) provides an illustration that this phenomenon does not take place on a constant basis; thus, the issue of the broader conditions under which it occurs is subject to discussion.

The research paper by Guillouzic et al. (1999) proves the similar phenomenon, i. e. the possibility to approximate the differential equation with insignificant argument retardation to the differential equation without any retardation.

As far as it is known, the phenomenon of dichotomy as regards the differential equations was initially discussed in the research papers written by Aulbach et al. (1994) and Aulbach and van Minh (1996). The overview of further results is presented, for instance, in the research paper by Berezansky and Braverman (2006).

Zheentaeva (2017) illustrated that using the differential equations with small argument retardation and the technique of splitting the solution space, it is possible to enable their transformation into the systems of operatordifference equations with preservation of their specifics. Thus, the new conditions ensuring the existence of special (slowly changing) solutions are obtained, their possession of approximating and asymptotically approximating properties is defined, as well as the asymptotic one-dimensional solution space of initial problems for linear scalar differential equations with insignificant argument retardation and corresponding operator- 
difference system of equations (special solutions correspond to the solutions with a slowly changing first component, and a relatively small second component).

In accordance with the concept used by Kenenbaeva et al. (2018), this means that the new uniform type of equations is defined for the functions with asymptotic properties; and the equivalent transformations produce different forms of equations within this type.

Also, Zheentaeva (2017) found the conditions for the systems of linear vector-matrix operator-difference equations and the corresponding linear vector-matrix differential equations with insignificant argument retardation, ensuring the existence of special solutions.

The purpose of this research paper is to study the new conditions that make sure that such special solutions possess approximating and asymptotically approximating properties, as well as the asymptotic one-dimensional space of solutions of the initial problems.

Section 2 outlines and introduces the required definitions and introduces the method of splitting the space of solution.

Section 3 reviews the known results.

Section 4 presents the newly found conditions making it possible for such special solutions to possess approximating and asymptotically approximating properties, and enabling the asymptotic one-dimensional space of solutions of the initial problems.

Section 5 provides examples of asymptotic factor-spaces of differential equation solutions.

\section{Required Definitions and Employed Method}

Let us denote as follows: $\boldsymbol{N}_{0}=\{0,1,2,3, \ldots\}, \boldsymbol{N}=\{1,2,3, \ldots\}, \boldsymbol{R}=(-\infty, \infty), \boldsymbol{R}_{+}=[0, \infty), \boldsymbol{R}_{++}=(0, \infty), I_{n^{-}} n \times n$ as identity matrix, $n \in \boldsymbol{N} ; C^{m(k)} D$ is the space of functions $u: D \rightarrow \boldsymbol{R}^{m}$ continuous with the derivatives till the order $k ; D$ is the domain in $\boldsymbol{R}, 0 \in D, m \in N, k \in \boldsymbol{N}_{0} ; C^{* m(k)} D$ is the sub-space of functions meeting the condition $u(0)=0 \in \boldsymbol{R}^{m} . m=1$ and $k=0$ shall be neglected.

D e f i n it i o 1 is for the category of equations Equa (Kenenbaeva et al., 2018). The objects in this category are sets \{non-empty sets of $X, Y$, and predicate $P(x)$ along $X$, the transformation $B: X \rightarrow Y$ \}. The solution of this equation $\{X, Y, P, B\}$ is such value of $y \in Y$, so that $(\exists x \in X)(P(x) \wedge(y=B(x))$. In particular, if $B$ is the identical representation, then the only task is to solve the equation of $P(x)^{\prime}$. Morphisms are such transformations of the sets $\{X, Y, P, B\}$, so that solutions thereof (as well as their absence) are recorded.

Transformation $B$ shall be considered as final.

For the purposes of the uniform representation of problems with continuous and discrete time, it shall be assumed that the argument of the desired function $t$ belongs to quite an ordered set $\Lambda$, possessing its smallest element (herein referred to as 0 ), but still not possessing any largest element. Normally, $\Lambda=\boldsymbol{R}_{+}$or $\Lambda=\boldsymbol{N}_{0}$ are employed.

This research paper discusses the initial problems only. Assuming that the initial problem always has some solution, then the solution is the only and global one, and, thus, it covers the entire set $\Lambda$; so, then the space of solutions of any particular dynamic system with the entry condition $\varphi$ may be regarded as the operator $W(t, \varphi): \Lambda \times \Phi \rightarrow Z$, with $\Phi$ being the topological space of the initial conditions, and $Z$ being the topological space of the solution values. In the case of $\Lambda=\boldsymbol{R}_{+}$, let us assume that $W(t, \varphi)$ is continuous with respect to $t$.

The following types of space shall be discussed herein, i. e. $\Phi$ and $Z$ : linear one-dimensional $(\boldsymbol{R})$; linear multidimensional $\left(\boldsymbol{R}^{d}\right)$; linear normed; uniform.

D e f i n i t i o $n$ 2. Let the function $\psi: \Lambda \rightarrow \boldsymbol{R}_{++}$be given. The following relation of equivalence to space $\Phi$ shall be referred to as the relation of asymptotic equivalence along $\psi$.

If $Z$ is a linear normed space, then

$$
\left(\varphi_{1} \sim \psi(t) \varphi_{2}\right) \Leftrightarrow\left(\lim \left\{\psi(t)\left\|W\left(t, \varphi_{1}\right)-W\left(t, \varphi_{2}\right)\right\|_{Z}: t \rightarrow \infty\right\}=0\right) .
$$

If $Z$ is a metric space, then

$$
\left(\varphi_{1} \sim \psi(t) \varphi_{2}\right) \Leftrightarrow\left(\lim \left\{\psi(t) \rho_{Z}\left(W\left(t, \varphi_{1}\right), W\left(t, \varphi_{2}\right)\right): t \rightarrow \infty\right\}=0\right) .
$$

At $\psi(t) \equiv l$, this definition may be extended onto even greater types of spaces. 
D e f i n i t i o n 3. The following relation of equivalence to space $\Phi$ shall be referred to as the relation of asymptotic equivalence:

If $Z$ is a linear normed space, then

$$
\left(\varphi_{1} \sim \varphi_{2}\right) \Leftrightarrow\left(\lim \left\{\left\|W\left(t, \varphi_{1}\right)-W\left(t, \varphi_{2}\right)\right\|_{Z:} t \rightarrow \infty\right\}=0\right) .
$$

If $Z$ is a metric space, then

$$
\left(\varphi_{1} \sim \varphi_{2}\right) \Leftrightarrow\left(\lim \left\{\rho_{Z}\left(W\left(t, \varphi_{1}\right), W\left(t, \varphi_{2}\right)\right): t \rightarrow \infty\right\}=0\right) .
$$

If $Z$ is a uniform space with the setmultiple entourages of diagonal $\Gamma_{Z}$, then

$$
\left(\varphi_{1} \sim \varphi_{2}\right) \Leftrightarrow\left(\forall V \in \Gamma_{Z}\right)\left(\exists t_{1} \in \Lambda\right)\left(\forall t>t_{1}\right)\left(\left(W\left(t, \varphi_{1}\right), W\left(t, \varphi_{2}\right)\right) \in V\right) .
$$

$\mathrm{L} \mathrm{e} \mathrm{m} \mathrm{m}$ a 1 . The introduced relation represents the correct relation of equivalence.

P r o o f. The reflexiveness and symmetry of relation are obvious. Let us prove transitivity.

Given are $\varphi_{1} \sim \varphi_{2}, \varphi_{2} \sim \varphi_{3}$. For (2) and according to the set $\varepsilon \in \boldsymbol{R}_{++}$, let us define such $t_{12}$, so that $\rho_{Z}\left(W\left(t, \varphi_{1}\right)\right.$, $\left.W\left(t, \varphi_{2}\right)\right)<\varepsilon / 2$ at $t>t_{12}$, and such $t_{23}$, so that $\rho_{Z}\left(W\left(t, \varphi_{2}\right), W\left(t, \varphi_{3}\right)\right)<\varepsilon / 2$ at $t>t_{23}$.

Therefore, at $t>t_{13}:=\max \left\{t_{12}, t_{23}\right\}$ the following is obtained:

$\rho_{Z}\left(W\left(t, \varphi_{1}\right), W\left(t, \varphi_{3}\right)\right) \leq \rho_{Z}\left(W\left(t, \varphi_{1}\right), W\left(t, \varphi_{2}\right)\right)+\rho_{Z}\left(W\left(t, \varphi_{2}\right), W\left(t, \varphi_{3}\right)\right)<\varepsilon / 2+\varepsilon / 2=\varepsilon$.

Consequently, (1) can be obtained from (2).

For (3), the axiom of composition of the diagonal entourages shall be considered: $\left(\forall V \in \Gamma_{Z}\right)\left(\exists V_{l} \in \Gamma_{Z}\right)\left(V_{l} V_{l}\right.$ $\subset V)$. Given $V \in \Gamma_{Z}$, it is possible to obtain, that such $V_{1}, t_{12}$, so that $\left(W\left(t, \varphi_{1}\right), W\left(t, \varphi_{2}\right)\right) \in \mathrm{V} 1$ at $\mathrm{t}>t_{12}$, and such $t_{23}$, so that $\left(W\left(t, \varphi_{2}\right), W\left(t, \varphi_{3}\right)\right) \in V_{l}$ at $t>t_{23}$.

Therefore, at $t>t_{13}:=\max \left\{t_{12}, t_{23}\right\}$ the following is obtained: $\left(W\left(t, \varphi_{1}\right), W\left(t, \varphi_{3}\right)\right) \in V_{1} V_{1} \subset V$.

Lemma is proven.

Exponential functions are commonly used as comparison functions. Consequently, it is required to introduce a separate

D e f i n i t i o n 4 . The following relation of equivalence to space $\Phi$ shall be referred to as the relation of $\lambda$ exponential asymptotic equivalence $\left(\lambda \in \boldsymbol{R}_{++}\right)$:

If $Z$ is a linear standardised space, then

$$
\left(\varphi_{1} \sim \varphi_{2}\right) \Leftrightarrow\left(\sup \left\{\left\|W\left(t, \varphi_{1}\right)-W\left(t, \varphi_{2}\right)\right\|_{z} \exp (\lambda t): t \in \Lambda\right\}<\infty\right) .
$$

If $Z$ is a metric space, then

$$
\left(\varphi_{1} \sim \varphi_{2}\right) \Leftrightarrow\left(\sup \left\{\rho_{Z}\left(W\left(t, \varphi_{1}\right), W\left(t, \varphi_{2}\right)\right) \exp (\lambda t): t \in \Lambda\right\}<\infty\right) .
$$

N o t e. Various research papers (Levenson, 1946) refer to the term `asymptotic equivalence` in another sense, i. e. as the proximity between the solutions of different equations with the same space of initial values. The reference herein is as follows:

$$
\left(W_{l}(t, \varphi) \approx W_{2}(t, \varphi)\right) \Leftrightarrow\left(\forall \varphi_{1} \in \Phi\right)\left(\exists \varphi_{2} \in \Phi\right)\left(\lim \left\{\left\|W_{l}\left(t, \varphi_{1}\right)-W_{2}\left(t, \varphi_{2}\right)\right\|: t \rightarrow \infty\right\}=0\right) .
$$

D e f i n it i o n 5. At $\Lambda=\boldsymbol{R}_{+}$the following equivalence relation in space $\Phi$ shall be referred to as the relation of Hausdorff asymptotic equivalence:

If $Z$ is a metric space, then $\left(\varphi_{1} \cong \varphi_{2}\right)$ is defined as follows: for any value in $\varepsilon \in \boldsymbol{R}_{++}$it is possible to find such value in $s \in \boldsymbol{R}_{+}$and such steadily and infinitely increasing continuous function $\vartheta:[s, \infty) \rightarrow \boldsymbol{R}_{+}$, so that $(\forall t \in[s, \infty))\left(\rho_{Z}\left(W\left(t, \varphi_{1}\right), W\left(\vartheta(t), \varphi_{2}\right)\right)<\varepsilon\right)$.

If $Z$ is a uniform space with multiple entourages of diagonal $\Gamma_{Z}$, then $\left(\varphi_{1} \cong \varphi_{2}\right)$ is defined as follows: for any value in $\varepsilon \in \Gamma_{Z}$ it is possible to find such value in $s \in \boldsymbol{R}_{+}$such steadily and infinitely increasing continuous function $\vartheta(t):[s, \infty) \rightarrow \boldsymbol{R}_{+}$, so that

$$
(\forall t \in[s, \infty))\left(\left(W\left(t, \varphi_{1}\right), W\left(\vartheta(t), \varphi_{2}\right)\right) \in \varepsilon\right) .
$$

$\mathrm{L} \mathrm{e} \mathrm{m} \mathrm{m}$ a 2 . The introduced relation represents the correct relation of equivalence. 
P r o o f. The reflexiveness of relation $\cong$ is obvious (assume $\vartheta(t) \equiv t)$.

Let us prove the symmetry. Let us assume that $\varphi_{1} \cong \varphi_{2}$. By definition, there is a function inverse to the function $\vartheta(t)$, being a continuous and steadily increasing function as well $\zeta(t): \boldsymbol{R}_{+} \rightarrow[s, \infty)$.

By substituting $\zeta(t)$ for $t$ in (6), the following is obtained:

$(\forall \zeta(t) \in[s, \infty))\left(\left(W\left(\zeta((t)), \varphi_{1}\right), W\left(\vartheta(\zeta(t)), \varphi_{2}\right)\right) \in \varepsilon\right) ;$

The condition of $\zeta(t) \geq s$ equals to the condition of $\vartheta(\zeta(t)) \geq \vartheta(s)$. Therefore,

$\left.(\forall t \in[\vartheta(s), \infty))\left(W(t), \varphi_{2}\right),\left(W\left(\zeta(t), \varphi_{1}\right)\right) \in \varepsilon\right) ; \varphi_{2} \cong \varphi_{1}$.

Let us validate transitivity. For the given $\varepsilon \in \Gamma_{Z}$, let us find such $\varepsilon_{l} \in \Gamma_{Z}$, so that $\varepsilon_{l}{ }^{\circ} \mathcal{E}_{l} \subset \varepsilon$. By condition, there exist such $s_{12}, s_{23}, \vartheta_{12}(t)$, and $\vartheta_{23}(t)$, so that

$$
\begin{aligned}
& \left(\forall t \in\left[s_{12}, \infty\right)\right)\left(\left(W\left(t, \varphi_{1}\right), W\left(\vartheta_{12}(t), \varphi_{2}\right)\right) \in \varepsilon_{1}\right), \\
& \left(\forall t \in\left[s_{23}, \infty\right)\right)\left(\left(W\left(t, \varphi_{2}\right), W\left(\vartheta_{23}(t), \varphi_{3}\right)\right) \in \varepsilon_{1}\right) .
\end{aligned}
$$

By substituting $\vartheta_{12}(t)$ for $t$ in (8), the following is obtained:

$$
\left(\forall \vartheta_{12}(t) \in\left[s_{23}, \infty\right)\right)\left(\left(W\left(\vartheta_{12}(t), \varphi_{2}\right), W\left(\vartheta_{23}\left(\vartheta_{12}(t)\right), \varphi_{3}\right)\right) \in \varepsilon_{1}\right) .
$$

The condition of $\vartheta_{12}(t) \geq s_{23}$ equals to the condition of $t \geq \zeta_{12}\left(s_{23}\right)$. Therefore, the relation (9) gets rewritten in the way as follows, and including the composition of functions:

$$
\left(\forall t \in\left[\zeta_{12}\left(s_{23}\right), \infty\right)\right)\left(W\left(\vartheta_{12}(t), \varphi_{2}\right),\left(W\left(\left(\vartheta_{23} \vartheta_{12}\right)(t), \varphi_{3}\right)\right) \in \varepsilon_{1}\right) .
$$

If $s_{13}=\max \left\{s_{12}, \zeta_{12}\left(s_{23}\right)\right\}$ is chosen, then the following is obtained from (7) and (10):

$\left(\forall t \in\left[s_{13}, \infty\right)\right)\left(\left(\left(W\left(t, \varphi_{1}\right), W\left(\vartheta_{12}(t), \varphi_{2}\right)\right) \in \varepsilon_{1}\right) \wedge\left(W\left(\vartheta_{12}(t), \varphi_{2}\right),\left(W\left(\left(\vartheta_{23} \vartheta_{12}\right)(t), \varphi_{3}\right)\right) \in \varepsilon_{1}\right)\right)$.

From here it is possible to obtain: $\left(\forall t \in\left[s_{13}, \infty\right)\right)\left(\left(W\left(t, \varphi_{1}\right), W\left(\left(\vartheta_{23} \vartheta_{12}\right)(t), \varphi_{3}\right)\right) \in \varepsilon_{1}{ }^{\circ} \varepsilon_{l}\right)$.

Transitivity is proven. Lemma is proven.

The asymptotic quotient space of Hausdorff space shall be referred to as $\Phi^{*=}$.

Let us consider the case, when all spaces are linear normalized, and space $\Phi$ is much larger than space $Z$, and space $\Phi_{0}$ represents certain (full) sub-space of space $\Phi$. Let us introduce

D e f i n i t i o n 6. Solutions to $W(t, \varphi), \varphi \in \Phi_{0}$, are referred to as special solutions, if

1) $\left(\forall t_{1} \in \Lambda\right)\left(\forall z_{1} \in Z\right)\left(\exists \varphi_{1} \in \Phi_{0}\right)\left(W\left(t_{1}, \varphi_{1}\right)=z_{l}\right)$;

2) $(\exists \lambda>0)\left(\forall \varphi_{1}, \varphi_{2} \in \Phi_{0}\right)\left(\forall t_{1}, t_{2} \in \Lambda\right)$

$$
\left(\left\|W\left(t_{1}, \varphi_{1}\right)-W\left(t_{1}, \varphi_{2}\right)\right\| \geq\left\|W\left(t_{2}, \varphi_{1}\right)-W\left(t_{2}, \varphi_{2}\right)\right\| \exp \left(-\lambda\left|t_{1}-t_{2}\right|\right)\right) .
$$
$\Phi)$.

Consequently, space $\Phi_{0}$ for special functions possesses dimensions of space $Z$ (as opposed to the entire space

The initial condition $\varphi_{1} \in \Phi_{0}$, existing and only available by virtue of the conditions 1) and 2), shall be referred to as $\gamma\left(t_{1}, z_{1}\right): \Lambda \times Z \rightarrow \Phi_{0}$.

D e f i n i t i o n 7. Special solutions are called approximating, if for any solution $W(t, \varphi), \varphi \in \Phi$, there exists the limit $\Gamma(\varphi):=\lim \{\chi(t, W(t, \varphi)): t \rightarrow \infty\}: \Phi \rightarrow \Phi_{0}$.

D e f i n i t i o $\mathrm{n}$ 8. Special solutions are called asymptotically approximating, if for any solution $W(t, \varphi), \varphi \in \Phi$, there exists the limit $\lim \{W(t, \varphi)-W(t, \Gamma(\varphi)): t \rightarrow \infty\}=0$, and $\lambda_{1}$ is exponentially asymptotically approximating $\left(\lambda_{l}>\lambda\right)$, if for any solution $W(t, \varphi), \varphi \in \Phi$ the following is valid: $\sup \left\{\|W(t, \varphi)-W(t, \Gamma(\varphi))\|_{z} \exp \left(\lambda_{l} t\right): t \in \Lambda\right\}<\infty$.

If $Z=\boldsymbol{R}^{d}$ (finite dimensional), and space $\Phi$ is infinite dimensional, then this phenomenon is referred to as asymptotic finite dimension of the solution space $W(t, \varphi), \varphi \in \Phi$, or, pursuant the wording proposed by the author, there observed an asymptotic decrease in dimension of the solution space.

For the linear dynamic systems (written as $\left.W(t, \varphi) \equiv W_{l}(t) \varphi, \varphi \in \Phi\right)$, these definitions are adjusted accordingly. The sub-space $\Phi_{0}$ implies its linear nature, and instead of it its basis is taken into account. 
Let us consider a differential equation with retarded argument:

$$
u^{\prime}(t)=P(t) u(t-h)\left(h \in \boldsymbol{R}_{++}\right), t \in \boldsymbol{R}_{+},
$$

where $u(t)$ is the unknown scalar or $m$-vector function, and $P(t)$ is the given continuous scalar or $m$-vector function respectively, with the initial condition:

$$
u(t)=\varphi(t), t \in[-h, 0],
$$

where $\varphi(t)$ is the given continuous scalar or m-vector function.

In the scalar case, if the function $u(t)$ possesses a physical dimension of length $(L)$, then $u$ ' $(t)$ has a physical dimension of $(L / T)$, with $T$ being time; thus, the function $P(t)$ has a physical dimension of $(1 / T)$. Consequently, the multiplication of $P(t) h$ is a dimensionless value; and estimates of the norm for this value, defining the presence of properties in Definitions 5-7, represent the absolute constants, but the internal interval representations of the range of its possible values are absolute domains.

It is common knowledge, that the initial problem (11)-(12) is equivalent to the following system of equations for functions $U_{n}(t) \in C^{m(1)}[-h, 0], n \in N_{0}$ :

$$
\begin{gathered}
U_{0}(t)=S_{0}(\varphi(\cdot))(t):=\varphi(0)+\int_{-h}^{t} P(s+h) \varphi(s) d s \\
U_{n+1}(t)=S_{n}\left(U_{n}(\cdot)\right)(t):=U_{n}(0)+\int_{-h}^{t} P(s+n h+h) U_{n}(s) d s, n \in \boldsymbol{N}_{0} .
\end{gathered}
$$

For the purposes of compliance with Definition 1, let us add the final ratio $z(t)=u(t), t \in \boldsymbol{R}_{+}$to (11)-(12), and the final formula $z(t)=U_{n}(t+n h+h)(n h \leq t<n h+h), n \in N_{0}$ shall be added to (13).

Aulbach et al. (1994) proposed the following method: let us consider the space $C^{m(1)}[-h, 0]=\boldsymbol{R}^{m} \times C^{m(1)}[-h, 0]$ as Cartesian product of space of functions-constants equal to $\boldsymbol{R}^{m}$, and the space $\Omega=C^{* m(1)}[-h, 0]$, namely $U_{n}(t)=x_{n}+y_{n}(t)$.

Let us denote that $P_{n}(\tau)=P(\tau+n h+h)$.

Consequently, the shift operators equal to the value of $h$ in (13) shall be given in the following way:

$$
\begin{gathered}
x_{n+1}+y_{n+1}(t)=S_{n}\left(x_{n}+y_{n}(\cdot)\right)(t)=x_{n}+y_{n}(0)+\int_{-h}^{t} P_{n}(s)\left(x_{n}+y_{n}(s)\right) d s= \\
=x_{n}+\int_{-h}^{0} P_{n}(s)\left(x_{n}+y_{n}(s)\right) d s+\int_{0}^{t} P_{n}(s)\left(x_{n}+y_{n}(s)\right) d s= \\
=\left(\int_{-h}^{0} P_{n}(s) d s+I_{m}\right) x_{n}+\int_{-h}^{0} P_{n}(s) y_{n}(s) d s+\int_{0}^{t} P_{n}(s) d s x_{n}+\int_{0}^{t} P y_{n}(s) d s .
\end{gathered}
$$

Let us denote (a number or matrix) $a_{n}:=\int_{-h}^{0} P_{n}(s) d s+I_{m}$, (either linear or vector functional) $b_{n} y(\cdot):=$ $\int_{-h}^{0} P_{n}(s) y(s) d s$, (either scalar or matrix-function) $\mathrm{c}_{n}(t):=\int_{0}^{t} P_{n}(s) d s$,

(operator of the function corresponding in dimension) $d_{n} y(\cdot)(t):=\int_{0}^{t} P_{n}(s) y(s) d s$.

Then, the system in (14) is modified as follows:

$x_{n+1}=a_{n} x_{n}+b_{n} y_{n}(\cdot)$,

$$
y_{n+1}(t)=\mathrm{c}_{n}(t) x_{n}+d_{n} y_{n}(\cdot)(t), n \in N_{0},
$$

with the following final transformation:

$z(t)=x_{n}+y_{n}(t+n h+h) \quad(n h \leq t<n h+h), n \in \mathbf{N}_{0}$.

This kind of transformation extends the equation types which could have asymptotic properties, and makes it possible to obtain new results for the known types of equations.

\section{Review of Known Results}

In relation to the equations in (11)-(12) in the scalar case, the results obtained by many authors, such as Myshkis (1972) and Driver et al. (1973), prove that for the absolute constant $\Delta=p_{0} h, p_{0}:=\|P\|_{\boldsymbol{R}_{+}}$from 


$$
\Delta<\frac{1}{e}=0.367 \ldots
$$

(optimal estimate), and there exist asymptotically approximating special solutions.

In order to generate the absolute domain extending the estimate in (16), let us consider the method of splitting the solution space as proposed by Zheentaeva (2017).

Let $\Omega$ be some linear normalized space. Let us consider four sequences: $a_{n} \in \boldsymbol{R}$; linear functionals $b_{n}: \Omega \rightarrow \boldsymbol{R}$; linear operators $c_{n}: R \rightarrow \Omega ; d_{n}: \Omega \rightarrow \Omega, n \in N_{0}$, and the system of operator-difference equations:

$$
x_{n+1}=a_{n} x_{n}+b_{n} y_{n}, y_{n+1}=c_{n} x_{n}+d_{n} y_{n}, n \in \mathbf{N}_{0}, x_{n} \in R, y_{n} \in \Omega \text {. }
$$

Let us assume that there exist such intervals as $A=\left[a_{-}, a_{+}\right]$, and 'general interval' bounded linear operators $B$, $C, D$, so that

$\left(\forall n \in \boldsymbol{N}_{0}, x \in \boldsymbol{R}, y \in \boldsymbol{\Omega}\right)\left(a_{n} \in A ; b_{n} y \in B y, c_{n} x \in C x, d_{n} y \in D y\right)$.

Let us denote a ball: $S(v):=\{y \in \Omega:\|y\| \Omega \leq v\}$.

$\mathrm{T} h$ e or e $\mathrm{m} 1$. If there exists such a number $w>0$, that

1) $\left.\eta_{-}:=[A+B S(w)]_{-}>0 ; 2\right)\|C+D S(w)\|_{\Omega} \leq w \eta_{-}$,

then there exists such (special) solution $\left\{X_{n}, Y_{n}\right\}$ to the system in (17), so that

$$
(\forall n \in N)\left(X_{n} \geq \eta_{-}^{n} ;\left\|Y_{n}\right\|_{\Omega} \leq w X_{n}\right) .
$$

Given the values of $b:=\|B\|_{\Omega,} c:=\|C\|_{\Omega}, d:=\|D\| \Omega$, the conditions of Theorem 1 are modified in the following way: 1) $\eta_{-}:=a_{-}-w b>0$; 2) $c+w d \leq w \eta_{-}$. Additionally, it needs to be noted that with the linear substitutions of variables, the values $a_{\rightarrow} a_{+}, \beta$ : $=b c, d$ become invariants, and without any loss of generality, it is assumed that $b=1$.

T h e o r e m 2. If 1) $\left.d<a_{-} ; 2\right)\left(a_{-}-d\right)^{2}>4 \beta$, then the following conditions of Theorem 1 are met: 1) and 2). It makes it possible to consider as follows:

$$
w=\frac{1}{2}\left(\left(a_{-}-d\right)-\sqrt{\left(a_{-}-d\right)^{2}-4 \beta}\right), \eta_{-}=\frac{1}{2}\left(a_{-}+d+\sqrt{\left(a_{-}-d\right)^{2}-4 \beta}\right) .
$$

The examples prove that these conditions are optimal.

Th e o r e m 3. If $\|A D-\mathrm{BC}\|_{\Omega}<\eta_{-}{ }^{2}$ (consequently, $\left.\omega_{0}:=\left(a_{+} d+\beta\right) q_{-}{ }^{-2}<1\right)$, then for any solution $\left\{x_{n}\right.$, $\left.y_{n}\right\}$ and for the special solution $\left\{X_{n}, Y_{n}\right\}$ as defined in Theorem 1, there exists the limit as follows:

$$
\gamma\{x, y\}:=\lim \left\{\frac{x_{n}}{x_{n}}: n \rightarrow \infty\right\},
$$

namely, the special solutions are approximating.

$\mathrm{T} h$ e or e $\mathrm{m}$ 4. If the conditions of Theorem 3 are met, and

$$
\omega_{0}\left(a_{+}+w\right)<1,
$$

then for any solution $\{x, y\}$ and for the special solution $\{X, Y\}$ as defined in Theorem 1, the ratio as follows shall be of relevance:

$$
\lim \left\{x_{n}-\gamma\{x, y\} X_{n}: n \rightarrow \infty\right\}=0,
$$

namely, the special solutions are asymptotically approximating.

These estimates were applied to the equations (11)-(12) in the scalar case under the assumption that $\leq P(t) \leq p_{+}\left(p_{-} \leq 0<p_{+} ;\left|p_{-}\right| \leq p_{+}\right)$, and in compliance with the system (15). For the space $\Omega=C^{*(1)}[-h, 0]$ there was introduced the standard condition $\|y\|_{\Omega}:=\sup \{|y(t) / t|:-h \leq t<0\}$. Therefore, $|y(t)| \leq|| y|| \Omega|t|$. The notations were introduced (dimensionless values) $\Delta_{-}:=p_{-} h, \Delta_{+}:=p_{+} h$.

From here, the following estimates for constants were obtained:

$$
\begin{aligned}
{\left[a_{-}, a_{+}\right] } & =\left[1+p_{-} h, 1+p_{+} h\right]=\left[1+\Delta_{-}, 1+\Delta_{+}\right] ; \\
b & =\sup \int_{-h}^{0}|P(s)| \cdot|s| d s \leq \frac{1}{2}\|P\| h^{2} ;
\end{aligned}
$$




$$
\begin{gathered}
c=\sup \left\{\left|\int_{0}^{t} P(s) d s\right| t^{-1}\right\} \leq\|P\| ; \mathrm{d}=\sup \left\{\left|\int_{0}^{t} P(s)\right| s|d s| t^{-1}\right\} \leq \frac{1}{2}\|P\| h=\frac{1}{2} \Delta_{+} . \\
\beta=b c=\frac{1}{2} \Delta_{+}{ }^{2} .
\end{gathered}
$$

The validating computations in accordance with the equations in Theorems 2 and 3 produced the absolute domain, for which the special solutions of the equation in (11) appear asymptotically approximating:

$\mathrm{T}$ h e o r e m 5 . This absolute domain contains the values of $\Delta_{-}=-0.14 ; \Delta_{+}=0.38$ or $\Delta_{-}=-0.10 ; \Delta_{+}=0.40$ or $\Delta_{-}=-0.05 ; \Delta_{+}=0.42$.

These results extended the area defined in (16).

It is noteworthy, that the validating computations for another type of the differential equations theory problems with delay were used by Lessard and Mireles James (2020).

\section{Conditions for Availability of Special Solutions of Two-Term Linear Differential Equations with Delay}

Let us consider a scalar differential equation with a retarded argument:

$$
u^{\prime}(t)=Q(t) u(t)+P(t) u(t-h)\left(h \in \boldsymbol{R}_{++}\right), t \in \boldsymbol{R}_{+},
$$

where, in addition to (11), $Q(t)$ is the set continuous function meeting the condition of $q_{-} \leq Q(t) \leq q_{+}$.

Let us introduce the following notation: $E(\sigma, \tau)=\exp \left(\int_{\sigma}^{\tau} Q(v) d v\right)$. Thus,

$$
u(t)=E(0, t) u(0)+\int_{0}^{t} E(s, t) P(s) u(s-h) d s, t \in[0, h] .
$$

Let us introduce the notation $E_{n}(\sigma, \tau)=\exp \left(\int_{\sigma}^{\tau} Q(v n h+h) d v\right)$.

Using the notations as those in (14), and the equation as in (24), we obtain as follows for the equation (23):

$$
\begin{gathered}
x_{n+1}+y_{n+1}(t)=S_{n}\left(x_{n}+y_{n}(\cdot)\right)(t)=E_{n}(0, t)\left(x_{n}+y_{n}(0)\right)+ \\
+\int_{-h}^{t} E_{n}(s, t) P_{n}(s)\left(x_{n}+y_{n}(s)\right) d s= \\
=E_{n}(0, t) x_{n}+\int_{-h}^{0} E_{n}(s, t) P_{n}(s)\left(x_{n}+y_{n}(s)\right) d s+ \\
+\int_{0}^{t} E_{n}(s, t) P_{n}(s)\left(x_{n}+y_{n}(s)\right) d s=x_{n}+\left(E_{n}(0, t)-1\right) x_{n}+ \\
+\int_{-h}^{0} E_{n}(s, 0) P_{n}(s)\left(x_{n}+y_{n}(s)\right) d s+\int_{-h}^{0}\left(E_{n}(s, t)-E_{n}(s, 0)\right) P_{n}(s)\left(x_{n}+y_{n}(s)\right) d s+ \\
+\int_{0}^{t} E_{n}(s, t) P_{n}(s)\left(x_{n}+y_{n}(s)\right) d s .
\end{gathered}
$$

Let us equate:

$$
\begin{gathered}
x_{n+1}=x_{n}+\int_{-h}^{0} E_{n}(s, 0) P_{n}(s)\left(x_{n}+y_{n}(s)\right) d s \\
y_{n+1}(t)=\left(E_{n}(0, t)-1\right) x_{n}+\int_{-h}^{0}\left(E_{n}(s, t)-E_{n}(s, 0)\right) P_{n}(s)\left(x_{n}+y_{n}(s)\right) d s+ \\
+\int_{0}^{t} E_{n}(s, t) P_{n}(s)\left(x_{n}+y_{n}(s)\right) d s
\end{gathered}
$$

Therefore, by denoting

$$
a_{n}:=1+\int_{-h}^{0} E_{n}(s, 0) P_{n}(s) d s, b_{n} y(\cdot):=\int_{-h}^{0} E_{n}(s, 0) P_{n}(s) y(s) d s,
$$




$$
\begin{gathered}
c_{n}:=\left(E_{n}(0, t)-1\right)+\int_{-h}^{0}\left(E_{n}(s, t)-E_{n}(s, 0)\right) P_{n}(s) d s+\int_{0}^{t} E_{n}(s, t) P_{n}(s) d s, \\
d_{n} y(\cdot):=\int_{-h}^{0}\left(E_{n}(s, t)-E_{n}(s, 0)\right) P_{n}(s) y(s) d s+\int_{0}^{t} E_{n}(s, t) P_{n}(s) y(s) d s,
\end{gathered}
$$

we obtain the system of operator-difference equations as in (17), and equivalent to (23).

Let us estimate:

$$
\begin{gathered}
a_{n} \in 1+h\left[p_{-}, p_{+}\right] \exp \left[h q_{-}, h q_{+}\right]=1+h\left[p_{-}, p_{+}\right] \exp \left(h q_{+}\right), \\
a_{-}=1+h p_{-} \exp \left(h q_{+}\right), a=1+h p_{+} \exp \left(h q_{+}\right) ; \\
\left|b_{n} y(\cdot)\right| \leq \int_{-h}^{0} \exp \left[h q_{-}, h q_{+}\right]\left[p_{-}, p_{+}\right]\|y\|_{\Omega}|s| d s=\exp \left[h q_{-}, h q_{+}\right]\left[p_{-}, p_{+}\right]\|y\|_{\Omega^{2}} h^{2} / 2, \\
b=\exp \left(h q_{+}\right)\|P\| h^{2} / 2 ; \\
\left|c_{n}\right| \leq\left|E_{n}(0, t)-1\right|+\int_{-h}^{0}\left|E_{n}(s, t)-E_{n}(s, 0)\right| \cdot\|P\| d s+\int_{0}^{t} E_{n}(s, t)\|P\| \cdot|d s| \leq \\
\leq \exp \left(h q_{+}\right)\|Q\| \cdot|t|+\int_{-h}^{0} \exp \left(h q_{+}\right)\|Q\| \cdot|t| \cdot\|P\| d s+\int_{0}^{t} \exp \left(h q_{+}\right)\|P\| \cdot|d s|= \\
=\exp \left(h q_{+}\right)(\|Q\| \cdot\|P\| h+\|P\|)|t|, \\
c=\exp \left(h q_{+}\right)(\|Q\| \cdot\|P\| h+\|P\|), \\
\left|d_{n} y(\cdot)\right| \leq \int_{-h}^{0}\left|E_{n}(s, t)-E_{n}(s, 0)\right| \cdot\|P\| \cdot\|y\|_{\Omega}|s| d s+\int_{0}^{t} E_{n}(s, t)\|P\| \cdot\|y\|_{\Omega}|s| \cdot|d s| \leq \\
\leq\left(\int_{-h}^{0} \exp \left(h q_{+}\right)\|Q\| \cdot|t| \cdot|s| \cdot\|P\| d s+\int_{0}^{t} \exp \left(h q_{+}\right)\|P\| \cdot|s| \cdot|d s|\right)\|y\|_{\Omega}= \\
=\exp \left(h q_{+}\right)\left(\|Q\| \cdot\|P\| h^{2} t / 2+\|P\| t^{2} / 2\right)\|y\|_{\Omega},
\end{gathered}
$$

$d=\exp \left(h q_{+}\right)\left(\|Q\| \cdot\|P\| h^{2}+\|P\| h\right) / 2$.

Let us denote $\Theta:=\|Q\| h, \theta:=\exp \left(h q_{+}\right)$as the dimensionless values, therefore we obtain:

$a_{-}=1+\theta \Delta_{-}, \quad a=1+\theta \Delta_{+}, b=\theta \Delta_{+} h / 2, \quad c=\theta(\Theta+1)\|P\|$,

$$
\beta=b c=\theta^{2} \Delta_{+}{ }^{2}(\Theta+1) / 2, d=\theta \Delta_{+}(\Theta+1) / 2 .
$$

The validating computations in accordance with the equations in Theorems 2 and 3 produced an absolute coefficient domain, for which the special solutions of the equation in (23) appear asymptotically approximating. For instance:

$\mathrm{T}$ h e o r e $\mathrm{m}$ 6. This space contains the following values: $-1 \leq Q(t) h \leq-0.5,-0.36 \leq P(t) h \leq 0.36$.

\section{Asymptotic Quotient Spaces of Solutions of Differential Equations}

5.1. If the equation (11) or (23) has the asymptotically approximating special solution, and $P(t) \geq 0$ (and $Q(t) \geq$ 0 ), namely the special solutions are monotonous, then the asymptotic quotient space of solutions is finite dimensional, which means that there exists a phenomenon of asymptotic reduction in dimension of the solution space.

5.2. For many types of linear autonomous dynamic equations, there exists the following set (finite or computational, then $\left.\lim \left\{\operatorname{Re} \lambda_{k}: k \rightarrow \infty\right\}=-\infty\right)$ of the eigenvalues $\lambda_{1}, \lambda_{2}, \lambda_{3}, \ldots$ in the plane of the complex numbers, such as $\exp \left(\lambda_{k} t\right)$, representing the equation solution; thus, the general solution is presented as the sum as follows:

$$
W(t, \varphi)=\sum_{k} C_{k}(\varphi) \exp \left(\lambda_{k} t\right), t \in \boldsymbol{R}_{+},
$$

where $C_{k}$ are linear operators (and in the case of $\Phi=Z=\boldsymbol{R}$ are functionals) from the initial condition (the polynominal multipliers are yet to appear for multiple eigenvalues). 
In existence of such values $\lambda_{k}$, that $\operatorname{Re} \lambda_{k}<0$, there appears a phenomenon of asymptotic reduction in dimension of the solution space, and the space $\Phi^{*}$ is presented as follows:

$$
\sum\left\{\gamma_{k} \exp \left(\lambda_{k} t\right): \operatorname{Re} \lambda_{k} \geq 0\right\}
$$

The asymptotic quotient space $\Phi^{*=}$ of Hausdorff space may comprise the mathematic objects unknown previously.

5.3. For the solution described in the form (25), the space $\Phi^{*=}$ in scalar equation consists of five types:

- $\quad$ solutions with principal member $\gamma_{1} \exp \left(\lambda_{1} t\right), \lambda_{1}>0, \gamma_{1}>0$,

- $\quad$ solutions with principal member $\gamma_{1} \exp \left(\lambda_{1} t\right), \lambda_{1}>0, \gamma_{1}<0$,

- $\quad$ solutions with principal member $\gamma_{1} \exp \left(\lambda_{1} t\right), \operatorname{Re} \lambda_{1}>0, \operatorname{Im} \lambda_{1} \neq 0$,

- $\quad$ solutions with principal member $\gamma_{1} \exp \left(\lambda_{1} t\right), \operatorname{Re} \lambda_{1}=0, \operatorname{Im} \lambda_{1} \neq 0$,

- $\quad$ solutions with principal member $\gamma_{1} \exp \left(\lambda_{1} t\right), \operatorname{Re} \lambda_{1}<0$.

5.4. Let $\Phi=Z=\boldsymbol{R}^{2}$. Let us consider the unknown system of the two autonomous equations under the initial condition as follows: $x^{\prime}(t)=y(t), y^{\prime}(t)=x(t), x(0)=\varphi_{1}, y(0)=\varphi_{2}$;

$$
\begin{aligned}
& (x(t), y(t)) \equiv W\left(t, \varphi_{1}, \varphi_{2}\right)=\left(\left(\varphi_{1}+\varphi_{2}\right) / 2 \cdot \exp t+\left(\varphi_{1}-\varphi_{2}\right) / 2 \cdot \exp (-t),\right. \\
& \left.\left(\varphi_{1}+\varphi_{2}\right) / 2 \cdot \exp t+\left(\varphi_{2}-\varphi_{1}\right) / 2 \cdot \exp (-t)\right) .
\end{aligned}
$$

Here $\Phi^{*=}$ comprises three elements:

- $\quad$ equivalence type described by the solution $W(t, 0,0)=(0,0)$ (saddle);

- $\quad$ equivalence type described by the solution $W(t, 1,0)$;

- $\quad$ equivalence type described by the solution $W(t,-1,0)$.

The names of last two types are unknown. Thus, we propose the following:

- 'path functions without beginning` $\left\{(x, y)=(t, t): t \in \boldsymbol{R}_{+}\right\} ;\left\{(x, y)=(-t,-t): t \in \boldsymbol{R}_{+}\right\}$.

\section{Conclusion}

This research paper demonstrates that the method of splitting the solution space along with the method of validating computations provide new results for bifurcation of solutions of differential equations with insignificantly retarded argument. The proposed asymptotic quotient spaces give new representations of the solution spaces for initial problems of dynamic systems.

\section{References}

[1]. Aulbach B., \& Van Minh N. (1996). The concept of spectral dichotomy for linear difference equations II, Journal of Difference Equations and Applications, 2(3), 251-262.

[2]. Aulbach B., Van Minh N., \& Zabreiko P. P. (1994). The concept of spectral dichotomy for linear difference equations, Journal of Mathematical Analysis and Applications, 185, 275-287.

[3]. Berezansky L., \& Braverman E. (2006). On exponential dichotomy for linear difference equations with bounded and unbounded delay, Proceedings of the Conference on Differential and Difference Equations and Applications, 169-178.

[4]. Driver R. D. (1976). Linear differential systems with small delays, Journal of Differential Equations, 21(1), 148-166.

[5]. Driver R. D., Sasser D. W., \& Slater M. L. (1973). The Equation x' (t) $=a x(t)+b x(t-\tau)$ with Small Delay, The American Mathematical Monthly, 80(9), 990-995.

[6]. Guillouzic S., L'heureux I., \& Longtin A. (1999). Small delay approximation of stochastic delay differential equations, Physical Review E (Statistical Physics, Plasmas, Fluids, and Related Interdisciplinary Topics), 59(4), 3970-3982. 
[7]. Kenenbaeva G. M., Askar kyzy L., Beishebaeva J. K., \& Mamatzhanuulu E. (2018). Elements of equation categories, Bulletin of the Institute of Mathematics of the National Academy of Sciences of the Kyrgyz Republic, 1, 88-95.

[8]. Lessard J. P., \& Mireles James J. D. (2020). A functional analytic approach to validated numerics for eigenvalues of delay equations, Journal of Computational Dynamics, 7(1), 123-158.

[9]. Levinson N. (1946). The asymptotic behavior of system of linear differential equations, American Journal of Mathematics, 68, 1-6.

[10]. Myshkis, A. D. (1972). Linear differential equations with retarded argument. Moscow: Nauka.

[11]. Pankov P. S. (1977a). Asymptotic finiteness of the solution space for one type of systems with delay, Differential equations, 13(4), 455-462.

[12]. Pankov P. S. (1977b). An example of a linear uniform differential equation with delay that does not have a finite-dimensional exponentially stable solution space $t \rightarrow \infty$. Studies into integro-differential equations. Frunze: Ilim.

[13]. Zheentaeva Zh. K. (2017). Study of asymptotics in solutions of equations with small delay. Saarbrücken, Deutschland: LapLambertAcademicPublishing. 